\title{
Neurogranin in the CSF signals early Alzheimer disease and predicts disease progression
}

L evels of neurogranin in the cerebrospinal fluid (CSF) can be used to detect early synaptic dysfunction in Alzheimer disease (AD) and predict disease progression, according to two recent studies. The findings provide strong evidence that neurogranin could be used not only as a diagnostic marker, but also as a prognostic marker in $\mathrm{AD}$.

Previous work has shown that CSF levels of neurogranin-a postsynaptic protein expressed in the brain-are abnormally high in patients with $\mathrm{AD}$, highlighting its potential as a biomarker of the disease. An elevated level of neurogranin is thought to be a direct marker of synaptic dysfunction, which is linked to cognitive symptoms in AD. Measuring CSF levels of neurogranin could, therefore, provide more

information about the disease process that underlies cognitive decline in $\mathrm{AD}$ than do tau, phosphorylated tau and amyloid- $\beta_{1-42}$ $\left(\mathrm{A} \beta_{1-42}\right)$, the three 'core' biomarkers.

"This type of biomarker is highly desired, as synaptic dysfunction is an early and central pathogenetic mechanism in AD," explains Erik Portelius, the primary researcher on one of the studies. "Such a biomarker might serve as a direct link that enables us to monitor the progressive cognitive symptoms." Both new studies investigated this possibility by monitoring participants over time to determine whether CSF levels of neurogranin can predict changes in pathology and cognitive function.

Portelius and his colleagues, who were one of the first groups to identify the potential of neurogranin as a biomarker in 2010, analyzed a cohort of 378 participants who at recruitment were diagnosed as having $\mathrm{AD}$, mild cognitive impairment (MCI) or no cognitive impairment.

Baseline measurements of neurogranin in the CSF confirmed previous findings: levels were highest in patients with $\mathrm{AD}$ and higher than normal in participants with MCI. Among patients with

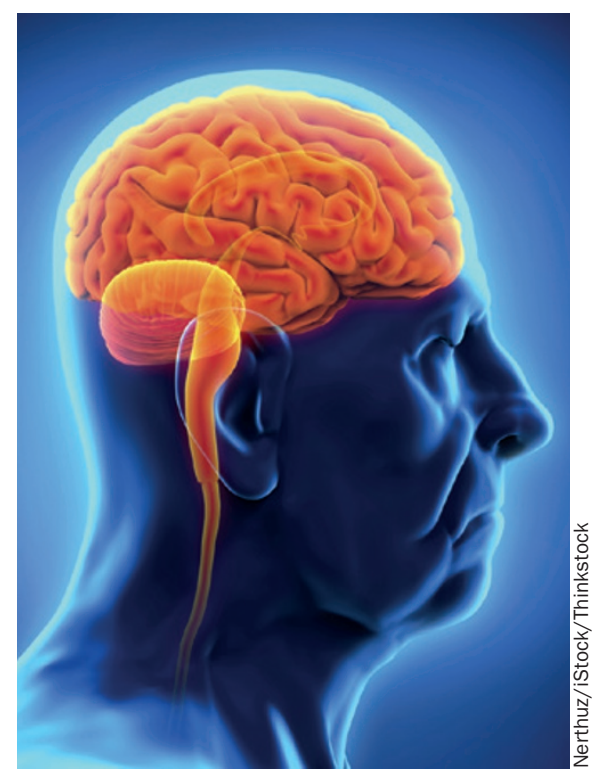

neurogranin levels also correlated strongly with those of total tau and phosporylated tau, but, in contrast to the findings of Portelius et al., not with levels of $A \beta_{1-42}$. Results from a follow-up period of 2 years demonstrated that higher CSF levels of neurogranin predicted progression from $\mathrm{MCI}$ to $\mathrm{AD}$ with similar accuracy as the core biomarkers.

Kester and colleagues also took a second measurement of CSF neurogranin levels at the end of the follow-up to enable comparison with levels at baseline. Surprisingly, they found no significant difference in any group except the controls who had no cognitive impairment.

"This finding indicates that CSF levels of neurogranin reflect very early synaptic loss within AD pathogenesis," explains Kester. "Neurogranin could, therefore, have a role in the diagnostic process, possibly in combination with the core CSF biomarkers, in identifying patients who are at risk of $\mathrm{AD}$, and in longitudinal monitoring of disease progression."

Portelius and Kester both say that an important next step is to identify whether neurogranin could be an effective biomarker in other neurodegenerative diseases. Either way, the combined evidence strongly indicates that neurogranin will prove valuable in the management of AD.

"We believe that CSF neurogranin will be highly important as a novel prognostic marker for prodromal AD and to monitor drug effects on synaptic degeneration in clinical trials of disease-modifying therapies for AD," concludes Portelius. Ian Fyfe

Original articles Portelius, E. et al. Cerebrospinal fluid neurogranin: relation to cognition and neurodegeneration in Alzheimer's disease. Brain doi:10.1093/brain/ awv267 | Kester, M. I. et al. Neurogranin as a cerebrospinal fluid biomarker for synaptic loss in symptomatic Alzheimer disease. JAMA Neurol. doi:10.1001/ jamaneurol.2015.1867 